\title{
MODELAGEM DA DISTRIBUIÇÃO DE DIÂMETROS UTILIZANDO AUTÔMATOS CELULARES E REDES NEURAIS ARTIFICIAIS
}

\author{
Daniel Henrique Breda Binoti ${ }^{1 *}$, Mayra Luiza Marques da Silva Binoti², \\ Helio Garcia Leite ${ }^{1}$, Antonilmar Araújo Lopes da Silva ${ }^{3}$, Ana Carolina Albuquerque ${ }^{1}$
}

*Autor para correspondência: danielhbbinoti@gmail.com

RESUMO: Esse estudo apresenta um modelo de distribuição diamétrica baseado em um modelo de Autômatos Celulares (AC) unidimensionais e redes neurais artificiais (RNA). Cada célula do AC proposto representa uma classe de dap, sendo o estado futuro previsto em função do estado atual dessa célula, do estado de suas quatro células vizinhas e de sua idade atual e futura. Como regra de evolução, utilizou-se uma RNA. A exatidão foi avaliada empregando: o procedimento estatístico proposto por Leite e Oliveira (2002); a relação entre frequência observada e estimada; e o realismo biológico do modelo construído. Dentre as redes treinadas, foram selecionadas as dez que representavam a evolução da distribuição diamétrica com maior exatidão. Dentre essas dez RNA, sete apresentaram valores estimados estatisticamente iguais aos observados $(p>0,01)$. O enfoque de modelagem proposto permite estimar distribuições diamétricas futuras com exatidão.

Palavras-chave: Inteligência artificial, distribuição diamétrica, eucalipto.

\section{AN APPROACH TO DIAMETER DISTRIBUTION MODELING USING CELLULAR AUTOMATA AND ARTIFICIAL NEURAL NETWORK}

\begin{abstract}
This study presents a diametric distribution model based on a one-dimensional cellular automata model (CA) and artificial neural network (ANN). Each cell of CA represents a dbh class, with the future state predicted in function of the present state of this cell, of the four neighboring cells and of its present and future age. An ANN was used as rule of evolution. Accuracy was evaluated by applying: statistical procedure proposed by Leite and Oliveira (2002); relation between observed and estimated frequency; and biological realism of the built model. Of the trained networks, were selected the ten representing the evolution of the diameter distribution with greater accuracy. Among these ten ANN, seven had estimated values statistically equal to observed $(p>0.01)$. The proposed modeling approach estimates accurately future diameter distributions.
\end{abstract}

Key words: Artificial intelligence, diameter distribution, eucalyptus.

\section{INTRODUÇÃO}

A projeção da distribuição diamétrica é essencial para o manejo de florestas cuja madeira é destinada a multiprodutos. Essa projeção pode ser feita empregando modelos que estimam a distribuição de diâmetros a partir de uma distribuição atual, ou de características do povoamento, permitindo simular desbastes e quantificar multiprodutos da madeira (CLUTTER et al., 1983; SIIPILEHTO et al., 2007). A característica comum nesse tipo de modelagem é a presença de uma função densidade de probabilidade $(f d p)$.

Diferentes tipos de distribuições estatísticas têm sido empregados para descrever a estrutura diamétrica de povoamentos florestais, como: Gama (NELSON, 1964), log-normal (BLISS; REINKER, 1965), Beta (CLUTTER; BENNETT, 1965), Johnson's SB (HAFLEY;
SCHUREUDER, 1977) e a distribuição Weibull (BAILEY; DELL, 1973). Desde 1973, a função Weibull é amplamente difundida e utilizada na área florestal (CAO, 2004; PALAHÍ et al., 2007).

Essas funções podem ser ajustadas pelos métodos dos momentos (FRAZIER, 1981), dos percentis (BAILEY et al., 1989), da máxima verossimilhança (FISHER, 1922), por combinação dos métodos dos momentos e dos percentis (BALDWIN JUNIOR; FEDUCCIA, 1987), empregando a meta heurística simulated annealing (ABBASI et al., 2006) ou empregando redes neurais artificiais (ABBASI et al., 2008).

A estimação da distribuição diamétrica futura pode ser feita empregando modelos de predição ou modelos de projeção. No primeiro caso, os parâmetros da função densidade de probabilidade são considerados como variáveis dependentes, em modelos de regressão onde as

\footnotetext{
${ }^{1}$ Universidade Federal de Viçosa - Viçosa, Minas Gerais, Brasil

${ }^{2}$ Universidade Federal dos Vales do Jequitinhonha e Mucuri - Diamantina, Minas Gerais, Brasil

${ }^{3}$ Cenibra - Belo Oriente, Minas Gerais, Brasil
}

Cerne, Lavras, v. 19, n. 4, p. 677-685, out./dez. 2013 
variáveis preditoras são características do povoamento (por exemplo, idade, diâmetros médio e máximo e índice de local). No segundo, os parâmetros observados numa idade atual também são incluídos como variáveis preditoras (CAO, 2004). Guimarães (1994) propôs um modelo de passo invariante, obtendo resultados compatíveis para a projeção da distribuição diamétrica a partir de quaisquer inventários florestais iniciais. A eficiência do modelo proposto por esse autor foi comprovada por Soares et al. (2007), com dados de povoamentos de eucalipto. Cao (2004) propôs os métodos da regressão de máxima verossimilhança e da regressão, utilizando a função de distribuição acumulativa para estimar os parâmetros da distribuição estatística de Weibull. Nogueira et al. (2005) propuseram um modelo de projeção da distribuição diamétrica por um sistema de equações, onde os parâmetros da função Weibull em uma idade futura foram correlacionados com esses mesmos parâmetros em uma idade atual, resultando em um sistema de projeção compatível. Adaptações e aplicações de diferentes modelos de distribuição de diâmetro podem ser encontrados em Burkhart (1971), Kangas e Maltamo (2000) e Nord-Larsen e Cao (2006).

Apesar da existência de diversas funções estatísticas, de diferentes métodos para ajuste dessas funções e de alternativas para a estimação de distribuições diamétricas futuras, o enfoque de modelagem tem sido o mesmo, com predomínio de uma das formas da função Weibull. Na maioria das vezes, os parâmetros dessa função têm sido correlacionados com atributos dos povoamentos por meio de regressão. $O$ presente estudo apresenta uma abordagem diferente daquelas usuais, envolvendo emprego de métodos de inteligência e de vida artificial ao invés da análise de regressão.

Técnicas da inteligência artificial, como redes neurais artificiais (SILVA, 2008), e de vida artificial, como autômatos celulares (MARTHEY et al., 2007), tem sido utilizadas em algumas aplicações na área florestal. Redes neurais artificiais (RNA) são processadores paralelamente distribuídos, compostos por unidades de processamento simples (neurônios artificiais), que armazenam conhecimento experimental (aprendizagem), tornando-o disponível para uso (generalização) (HAYKIN, 2001). Autômatos celulares (AC) são sistemas de interações locais, discretos no tempo e no espaço, compostos de células, onde o estado de cada célula é resultado de seu estado atual, da sua regra de transição e da sua interação com células vizinhas (ILACHINSKI, 2001). As células são componentes simples que interagem localmente e a complexidade em âmbito global advém de regras entre essas unidades mais simples. Diversos métodos podem ser utilizados como regras de transição, com destaque para RNA (GARZON, 1995).

A modelagem empregando AC tem sido feita em vários tipos de estudos, envolvendo fenômenos químicos (GERHARDT; SCHUSTER, 1989), crescimento vegetal (LINDEMAYER; ROZENBERG, 1976), crescimento de cristais (KESSLER et al., 1990), ecologia (PHIPPS, 1992), propagação de doenças infecciosas (SEGEL, 1999), dinâmica social (EPSTEIN; AXTELL, 1996) e incêndios florestais (BAK et al., 1990).

Neste estudo, objetivou-se construir e avaliar um modelo de autômatos celulares, com sua regra de evolução baseada em redes neurais artificiais, para a projeção da distribuição de diâmetros em povoamentos de eucalipto.

\section{MATERIAL E MÉTODOS}

\subsection{Dados}

Os dados utilizados nesse estudo foram obtidos de 400 parcelas retangulares permanentes de $340 \mathrm{~m}^{2}$, instaladas em povoamentos de híbridos de Eucalyptus grandis x Eucalyptus urophylla, na região Centro Oeste do Estado de Minas Gerais, Brasil. Esses povoamentos foram estabelecidos sob arranjo espacial de $3 \times 3 \mathrm{~m}$, com rotação regulatória de 7 anos, sendo a madeira utilizada para a produção de polpa de celulose de fibra curta. Nessa idade, a produtividade média nesses povoamentos variou de 25 nos piores locais a $50 \mathrm{~m}^{3} \cdot \mathrm{ha}^{-1} \cdot$ ano $^{-1}$ nos melhores. As medições de diâmetros foram efetuadas nas árvores com dap acima de $5 \mathrm{~cm}$, nas idades médias de 28, 40, 52 , 64 e 76 meses. O banco de dados foi separado em dois conjuntos de dados, sendo um para construção do modelo e o outro para sua validação a fim de que cada grupo de dados tenha igual variabilidade. Nessa separação, foi mantida uma frequência homogênea de dados por classe de capacidade produtiva.

\subsection{Modelo de Autômatos Celulares}

Para um modelo unidimensional de AC, o valor da $i^{a}$ célula no tempo $t$, denominado por $c_{i}(t)$, evolui de acordo com a regra $\mathrm{F}$ que é função do $c_{i}(t)$ e das outras células que se encontram dentro da extensão $r$ (para ambas as direções) de $c_{i}(t)$ (ILACHINSKI, 2001), isto é:

$C_{i}(t)=F\left(C_{i-r}(t-1), \ldots, C_{i+r}(T+1)\right)$ 
O AC proposto neste estudo é unidimensional, sendo que cada célula possui 4 vizinhos $(r=2)$. As células representam uma classe de diâmetro de $2 \mathrm{~cm}$ de amplitude, sendo que o estado de cada célula corresponde à probabilidade de ocorrência de árvores nesta classe.

Para a construção da regra de evolução do AC foram treinadas redes neurais artificiais, utilizando as seguintes variáveis de entrada: estado atual da célula, estado das células contidas na extensão da sua vizinhança e as idades atual e futura. Como variável de saída foi considerado o estado futuro da célula. Após a normalização dos dados foram testadas diferentes arquiteturas e tipos de redes, sendo selecionada aquela que melhor representava os dados. Foram testadas redes de função de base radial (RBF - Radial Basis Function), perceptrons de múltiplas camadas (MLP - Multilayer perceptron) e perceptrons de camada única (Perceptron), com diferentes arquiteturas, conforme Haykin (2001). A projeção da densidade (número de árvores por hectare) foi feita empregando RNA, tendo como variáveis de entrada a densidade atual, a idade atual e a idade futura. O software NeuroForest (2012) foi utilizado nessa etapa do estudo.

\subsection{Aplicação e validação do modelo AC}

O modelo de AC foi empregado para projetar a distribuição de diâmetros para todas as idades de medição. Gráficos da relação entre valores observados e estimados foram feitos para todas essas projeções e o procedimento estatístico proposto por Leite e Oliveira (2002) foi utilizado para avaliar a exatidão das projeções a $5 \%$ de significância.

\section{RESULTADOS E DISCUSSÃO}

\subsection{Regra de evolução}

Cerca de 50 redes diferentes foram ajustadas, escolhendo-se as dez que melhor representaram o realismo biológico envolvido na evolução da distribuição diamétrica. Essas dez redes foram utilizadas para o desenvolvimento do estudo, sendo suas principais características apresentadas na Tabela 1. Melhores resultados foram obtidos com as redes MLP (Tabela 1).

\subsection{Recuperação da distribuição diamétrica}

Com as dez melhores redes obtidas foi feita a projeção da distribuição diamétrica, empregando-se os dados das parcelas destinado à validação do modelo. Os resultados da aplicação do procedimento proposto por Leite e Oliveira (2002) são apresentados na Tabela 2. Esses resultados demonstram que as regras de evolução representaram a dinâmica do fenômeno estudado com eficiência e exatidão.

Escolheu-se uma parcela ao acaso para a simulação detalhada da dinâmica de evolução da distribuição de diâmetros (Tabela 3). Como exemplo, seja a célula correspondente a classe de dap $10 \mathrm{~cm}$ no tempo $t=0$

Tabela 1 - Principais características de dez redes neurais artificiais treinadas para avaliar a evolução da distribuição diamétrica de povoamentos de eucalipto.

Table 1 - Main characteristics of ten artificial neural networks trained to evaluate the evolution of diametric distribution of eucalypt stands.

\begin{tabular}{ccccccc}
\hline \multirow{2}{*}{ Identificação } & Arquitetura & $\begin{array}{c}\mathrm{N}^{\circ} \text { de neurônios } \\
\text { de entrada }\end{array}$ & $\begin{array}{c}\mathrm{N}^{\circ} \text { de neurônios } \\
\text { intermediários }\end{array}$ & $\begin{array}{c}\mathrm{N}^{\circ} \text { de neurônios } \\
\text { de saída }\end{array}$ & \multicolumn{2}{c}{ Função de ativação } \\
\cline { 6 - 8 } Rede-1 & MLP & 7 & 7 & 1 & Camada oculta & Camada de saída \\
Rede-2 & MLP & 7 & 9 & 1 & Exponencial & Logística \\
Rede-3 & MLP & 7 & 10 & 1 & Logística & Seno \\
Rede-4 & MLP & 7 & 12 & 1 & Logística & Identidade \\
Rede-5 & MLP & 7 & 10 & 1 & Logística & Logística \\
Rede-6 & MLP & 7 & 7 & 1 & Exponencial & Identidade \\
Rede-7 & MLP & 7 & 7 & 1 & Exponencial & Identidade \\
Rede-8 & MLP & 7 & 8 & 1 & Exponencial & Hiperbólica \\
Rede-9 & MLP & 7 & 6 & 1 & Logística & Hiperbólica \\
Rede-10 & MLP & 7 & 12 & Hiperbólica & Exponencial \\
\hline
\end{tabular}

Cerne, Lavras, v. 19, n. 4, p. 677-685, out./dez. 2013 
Tabela 2 - Resultados do procedimento estatístico proposto por Leite e Oliveira (2002), para as dez melhores RNA ( $\alpha=5 \%$ ), com o resultado da avaliação da hipótese $\mathrm{H}_{0}$ : A distribuição de diamétrica estimada é igual à projeção estimada.

Table 2 - Results obtained by applying the statistical procedure proposed by Leite and Oliveira (2002) for the best ten ANN ( $\alpha=$ $5 \%$ ), with evaluation result of hypothesis $H_{0}$ : The estimated diametric distribution is equal to the estimated projection.

\begin{tabular}{|c|c|c|c|c|c|c|c|c|c|c|}
\hline & Rede - 1 & Rede - 2 & Rede - 3 & Rede - 4 & Rede - 5 & Rede - 6 & Rede - 7 & Rede - 8 & Rede - 9 & Rede - 10 \\
\hline $\mathrm{F}(\mathrm{Ho})$ & $1,1780^{\mathrm{ns}}$ & $1,3505^{\mathrm{ns}}$ & $0,9479^{\mathrm{ns}}$ & $1,0291^{\mathrm{ns}}$ & $0,7727^{\text {ns }}$ & $0,4837^{\mathrm{ns}}$ & $0,5619^{\mathrm{ns}}$ & $1,5080^{\mathrm{ns}}$ & $0,7352^{\mathrm{ns}}$ & $0,7248^{\mathrm{ns}}$ \\
\hline $\mathrm{t}(\mathrm{e})$ & $0,6743^{\mathrm{ns}}$ & $1,3772^{\mathrm{ns}}$ & $0,3978^{\mathrm{ns}}$ & $1,1972^{\text {ns }}$ & $0,7198^{\mathrm{ns}}$ & $0,7234^{\mathrm{ns}}$ & $0,0159^{\text {ns }}$ & $0,7682^{\mathrm{ns}}$ & $0,6847^{\mathrm{ns}}$ & $0,6207^{\text {ns }}$ \\
\hline$r_{y_{j} y_{1}}>=1-|\bar{e}|$ & $\operatorname{sim}$ & $\operatorname{sim}$ & $\operatorname{sim}$ & $\operatorname{sim}$ & $\operatorname{sim}$ & $\operatorname{sim}$ & não & não & não & $\operatorname{sim}$ \\
\hline$r^{2}$ & 0,8775 & 0,9470 & 0,9050 & 0,9224 & 0,8927 & 0,9437 & 0,9034 & 0,8644 & 0,8826 & 0,9107 \\
\hline RMS & 0,0033 & 0,0015 & 0,0026 & 0,0023 & 0,0031 & 0,0015 & 0,0025 & 0,0032 & 0,0031 & 0,0020 \\
\hline Erro médio (e) & 0,0837 & 0,1357 & 0,0490 & $-0,1408$ & 0,0911 & $-0,0613$ & 0,0009 & 0,0636 & 0,0501 & 0,0479 \\
\hline
\end{tabular}

Tabela 3 - Probabilidade de ocorrência de árvores (estado) por classes de dap em cinco medições de uma parcela em um povoamento de eucalipto.

Table 3 - Probability of occurrence of trees (state) by dbh classes in five measurements of a plot in a eucalypt stand.

\begin{tabular}{|c|c|c|c|c|c|c|c|c|c|c|c|c|}
\hline Classe & 4 & 6 & 8 & 10 & 12 & 14 & 16 & 18 & 20 & 22 & 24 & 26 \\
\hline & \multicolumn{12}{|c|}{ Probabilidade observada } \\
\hline $\mathrm{t}=0$ & 0,00 & 0,02 & 0,11 & 0,54 & 0,32 & 0,01 & 0,00 & 0,00 & 0,00 & 0,00 & 0,00 & 0,00 \\
\hline $\mathrm{t}=1$ & 0,00 & 0,01 & 0,03 & 0,11 & 0,36 & 0,41 & 0,13 & 0,01 & 0,00 & 0,00 & 0,00 & 0,00 \\
\hline $\mathrm{t}=2$ & 0,00 & 0,01 & 0,01 & 0,05 & 0,15 & 0,35 & 0,35 & 0,08 & 0,01 & 0,00 & 0,00 & 0,00 \\
\hline$t=3$ & 0,00 & 0,01 & 0,01 & 0,03 & 0,10 & 0,23 & 0,36 & 0,18 & 0,04 & 0,01 & 0,00 & 0,00 \\
\hline \multirow[t]{2}{*}{$\mathrm{t}=4$} & 0,00 & 0,01 & 0,01 & 0,03 & 0,09 & 0,21 & 0,32 & 0,25 & 0,07 & 0,02 & 0,01 & 0,00 \\
\hline & \multicolumn{12}{|c|}{ Probabilidade estimada } \\
\hline $\mathrm{t}=0$ & - & - & - & - & - & - & - & - & - & - & - & - \\
\hline $\mathrm{t}=1$ & 0,00 & 0,02 & 0,01 & 0,11 & 0,33 & 0,43 & 0,10 & 0,01 & 0,00 & 0,00 & 0,00 & 0,00 \\
\hline$t=2$ & 0,00 & 0,01 & 0,01 & 0,06 & 0,15 & 0,38 & 0,29 & 0,08 & 0,01 & 0,00 & 0,00 & 0,00 \\
\hline $\mathrm{t}=3$ & 0,00 & 0,01 & 0,02 & 0,04 & 0,12 & 0,27 & 0,34 & 0,16 & 0,03 & 0,00 & 0,00 & 0,00 \\
\hline $\mathrm{t}=4$ & 0,00 & 0,01 & 0,03 & 0,03 & 0,09 & 0,18 & 0,31 & 0,23 & 0,09 & 0,03 & 0,00 & 0,00 \\
\hline
\end{tabular}

(idade $=27$ meses). Essa célula apresentava o valor (probabilidade) de 0,54 , sendo que suas vizinhas apresentavam os valores de 0,$02 ; 0,11 ; 0,32$ e 0,01 , representando, respectivamente, os valores das classes 6 , 8,12 e 14 . No instante $t=1$ (idade $=39$ meses), o valor dessa célula (célula correspondente a classe de dap 10 $\mathrm{cm}$ ) é de 0,11 . Esse resultado advém da aplicação da regra de transição sobre o estado atual do sistema no instante $t=0$. A aplicação da regra ocorre discretamente no tempo, o que pode ser observado para as outras classes da distribuição.

Cerne, Lavras, v. 19, n. 4, p. 677-685, out./dez. 2013
A RNA2 (Tabela 1) foi escolhida como sendo a mais representativa da evolução da distribuição de diâmetros. A partir dessa rede, foram projetadas 10 parcelas escolhidas ao acaso, dentre aquelas separadas para validação do modelo (Figura 1). A situação inicial da parcela 1 dessa figura condiz com uma situação típica dos povoamentos equiâneos com a distribuição tendendo a normal. A dinâmica de evolução tende a tornar a distribuição assimétrica à direita, em razão das árvores de maiores dimensões apresentarem maiores taxas de crescimento radial em relação às árvores menores (GUIMARÃES, 1994). 


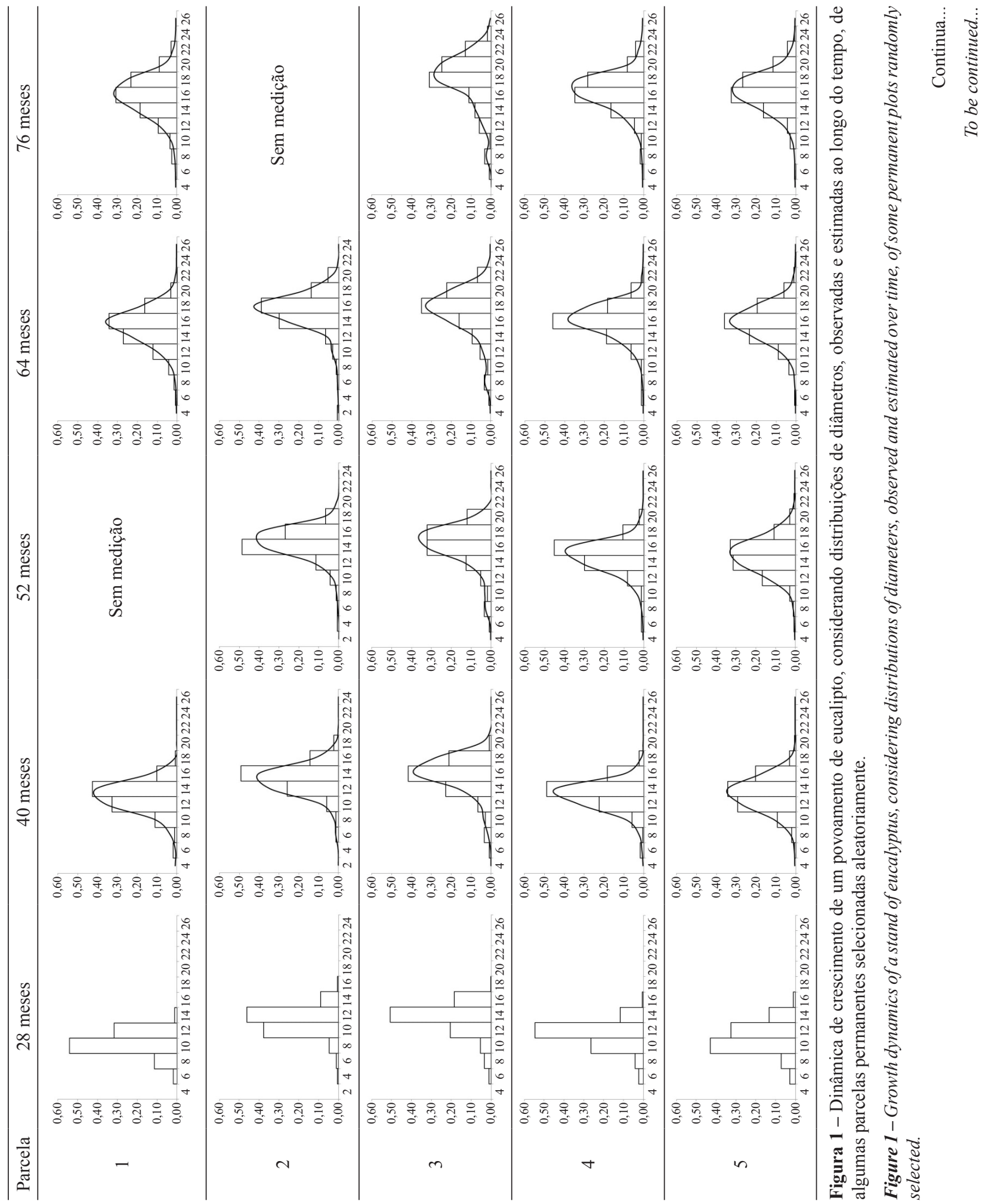

Cerne, Lavras, v. 19, n. 4, p. 677-685, out./dez. 2013 


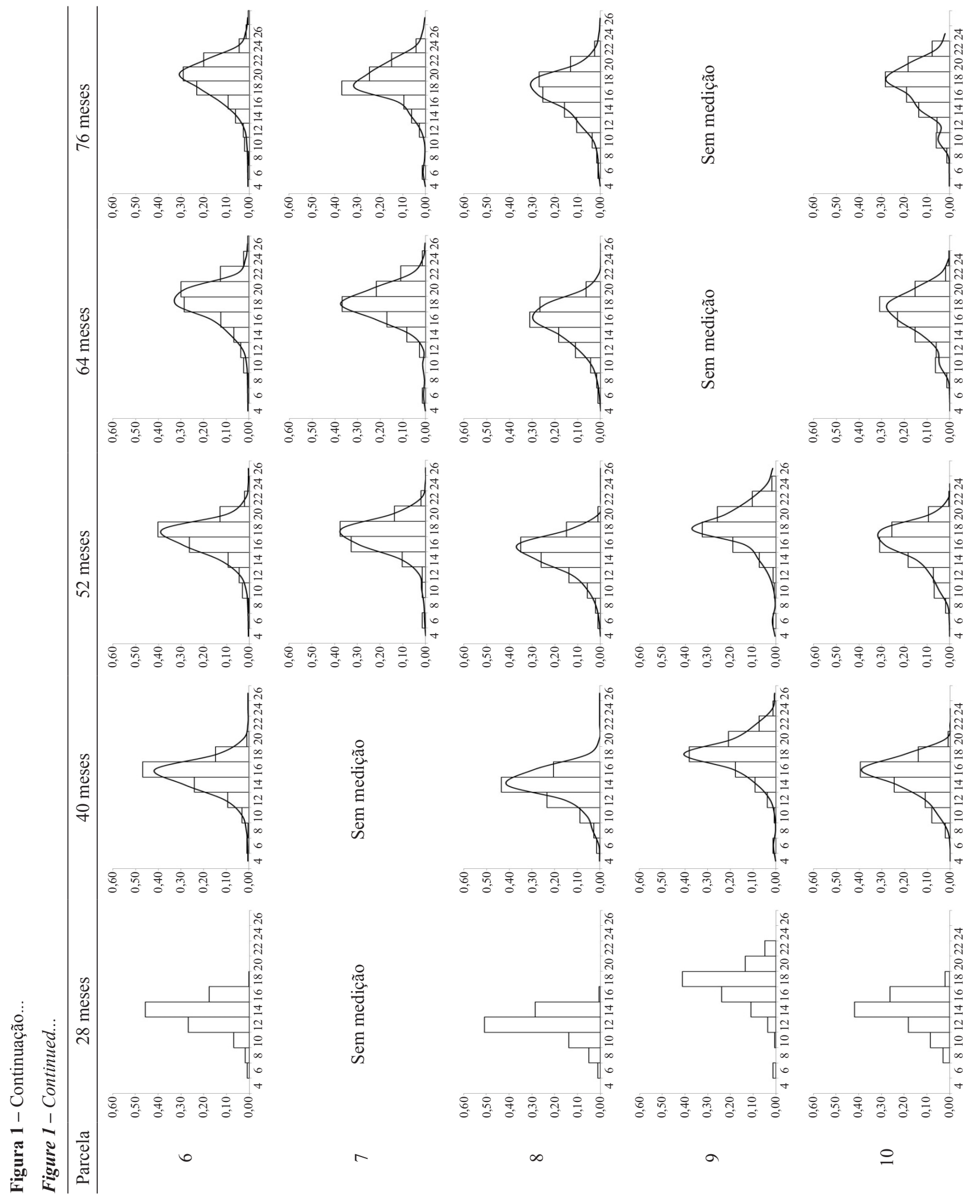

Cerne, Lavras, v. 19, n. 4, p. 677-685, out./dez. 2013 
A distribuição inicial da parcela 2, com frequência concentrada em três classes de dap, indica que a competição deve ter iniciado em idade mais avançada. As parcelas 3, 7 e 9 apresentam um típico caso de evolução da distribuição diamétrica de povoamentos equiâneos onde, devido a competição, o crescimento radial nas árvores suprimidas estagnou. As parcelas 4, 5, 6, 8 e 10 demonstram que diferentes relações entre as classes de dap podem ser descritas pela regra de evolução. O modelo construído foi capaz de descrever diferentes tendências de evolução na distribuição diâmetros.

A modelagem de distribuições de diâmetros em povoamentos de eucalipto tem sido feita com base em predição de parâmetros de uma distribuição estatística, empregando modelos de regressão. A função Weibull de dois parâmetros tem sido utilizada na maioria dos estudos por ser uma função flexível e pelo fato de seus parâmetros serem facilmente correlacionados com características dos povoamentos. Embora existam diferentes formas da função Weibull (MURTHY et al., 2004), na maioria das vezes essa função tem sido ajustada sem truncamentos à direita e à esquerda, pelo método da máxima verossimilhança e os modelos de distribuição de diâmetros envolvem conjuntos de equações que estimam os parâmetros da distribuição estatística utilizada. Cada uma das equações desses sistemas tem um erro associado, sendo a estimativa dos parâmetros da função estatística dependente da exatidão das equações que compõem o sistema.

A eficiência das estimativas geradas pelos modelos de distribuição de diâmetros usuais é dependente da qualidade dos dados utilizados e da qualidade do ajuste das equações que compõem o modelo. Em alguns casos, a frequência total estimada pelo modelo difere muito da frequência total observada ou estimada em nível de povoamento. Nesses casos, Clutter et al. (1983) recomendam que a diferença entre o somatório das frequências estimadas por classe de diâmetro e a frequência total prevista seja acrescida na última classe de diâmetro. Em povoamentos de eucalipto esta alternativa pode resultar em superestimação do volume por hectare. Outra alternativa consiste em redistribuir a diferença proporcionalmente às frequências estimadas por classe, resultando em estimativas de volume de maior exatidão.

No presente estudo, foi proposto um método alternativo para a modelagem de distribuições diamétricas que não envolve o uso de modelos de regressão para estimar a proporção de árvores por classe de diâmetro. A proposta consiste em associar um modelo de autômatos celulares e redes neurais artificiais. É uma abordagem simples que exige apenas distribuições de diâmetros observadas. Conforme resultado desse estudo, o enfoque de modelagem resulta em estimativas livres de tendenciosidade. Especialmente no caso de povoamentos submetidos a desbaste, ou povoamentos com distribuições erráticas, como aquelas indicadas na idade de 76 meses para as parcelas 3 e 10 (Figura 1), a metodologia proposta é sugestiva. Outra proposta eficiente para a modelagem de distribuições irregulares (erráticas) empregando a função Weibull foi apresentada por Zhang e Liu (2006). Embora não tenha sido apresentado por esses autores, a projeção de distribuições diamétricas a partir da função Weibull modificada também pode requerer o emprego modelos de regressão incluindo as variáveis independentes usuais (diâmetros médio e máximo, idade, índice de local etc.).

As estatísticas de precisão obtidas neste estudo são semelhantes àquelas obtidas em muitos modelos clássicos de distribuição de diâmetros o que demonstra a eficiência da metodologia proposta. Embora não tenha sido apresentada, a modelagem citada neste estudo pode ser empregada para povoamentos submetidos a qualquer tipo de desbaste e também para povoamentos inequiâneos. Conforme observado na Figura 1 (parcelas 3 e 10), a metodologia permite obter projeções descontinuas que podem ocorrer dependendo da mortalidade e do tipo de desbaste realizado. Isso normalmente não ocorre nos enfoques usuais, que geram distribuições continuas.

\section{CONCLUSÕES}

O modelo proposto neste estudo, baseado em autômatos celulares e em redes neurais artificiais, pode ser utilizado para a projeção da distribuição diamétrica de povoamentos equiâneos.

\section{REFERÊNCIAS}

ABBASI, B.; JAHROMIA, A. H. E.; ARKAT, J.; HOSSEINKOUCHACK, M. Estimating the parameters of Weibull distribution using simulated annealing algorithm. Applied Mathematics and Computation, New York, v. 183, n. 1, p. 85-93, 2006.

ABBASI, B.; RABELO, L.; HOSSEINKOUCHACK, M. Estimating parameters of the three-parameter Weibull distribution using a neural network. European Journal of Industrial Engineering, London, v. 2, n. 4, p. 428-445, 2008.

Cerne, Lavras, v. 19, n. 4, p. 677-685, out./dez. 2013 
BAILEY, R. L.; BURGAN, T. M.; JOKELA, E. J. Fertilized midrotation-aged slash pine plantations: stand structure and yield prediction models. Southern Journal of Applied Forestry, Washington, v. 13, n. 2, p. 76-80, 1989.

BAILEY, R. L.; DELL, T. R. Quantifying diameter distributions with the Weibull function. Forest Science, Bethesda, v. 19, n. 2, p. 97-104, 1973.

BAK, P.; CHEN, K.; TANG, C. A forest-fire model and some thoughts on turbulence. Physics Letters A, London, v. 147, n. 5/6, p. 297-300, 1990.

BALDWIN JUNIOR, V. C.; FEDUCCIA, D. P. Loblolly pine growth and yield prediction of managed West Gulf plantations. New Orleans: USDA, 1987. 27 p.

BLISS, C. I.; REINKER, K. A. A lognormal approach to diameter distributions in even-aged stands. Forest Science, Bethesda, v. 10, n. 3, p. 350-360, 1964.

BURKHART, H. E. Slash pine plantations yields estimates based on diameter distribution: an evaluation. Forest Science, Bethesda, v. 17, n. 4, p. 452-453, 1971.

CAO, Q. V. Predicting parameters of a Weibull function for modeling diameter distribution. Forest Science, Bethesda, v. 50, n. 5, p. 682-685, 2004.

CLUTTER, J. L.; BENNETT, F. A. Diameter distributions in old-field slash pine plantations. Georgia: Forest Research Council Report, 1965. 9 p. (Report, 13).

CLUTTER, J. L.; FORTSON, J. C.; PIENAAR, L. V.; BRISTER, R. G. H.; BAILEY, R. L. Timber management: a quantitative approach. New York: J. Willey, 1983. 333 p.

EPSTEIN, J. M.; AXTELL, R. Growing artificial societies: social science from the rottorn up. Cambridge: MIT, 1996. 224 p.

FISHER, R. A. On the interpretation of $\chi 2$ from contingency tables, and the calculation of P. Journal of the Royal Statistical Society, London, v. 85, n. 1, p. 87-94, 1922.

FRAZIER, J. R. Compatible whole-stand and diameter distribution models for loblolly pine plantations. 1981.

Cerne, Lavras, v. 19, n. 4, p. 677-685, out./dez. 2013
125 p. Thesis (Ph.D. in Forest Science) - Virginia Polytech Institute and State University, Blackburg, 1981.

GARZON, M. Models of massive parallelism: analysis of cellular automata and neural networks. Berlin: SpringerVerlag, 1995. 272 p.

GERHARDT, M.; SCHUSTER, H. A cellular automaton describing the formation of spatially ordered structures in chemical systems. Physica D: Nonlinear Phenomena, London, v. 36, n. 3, p. 209-221, 1989.

GUIMARÃES, D. P. Desenvolvimento de um modelo de distribuição diamétrica de passo invariante para prognose e projeção da estrutura de povoamentos de eucalipto. 1994. 178 f. Tese (Doutorado em Ciência Florestal) - Universidade Federal de Viçosa, Viçosa, 1994.

HAFLEY, W. L.; SCHREUDER, H. T. Statistical distributions for fitting diameter and height data in even-aged stands. Canadian Journal of Forest Research, Ottawa, v. 7, n. 3, p. 481-487, 1977.

HAYKIN, S. Redes neurais: princípios e prática. 2. ed. Porto Alegre: Bookman, 2001. 900 p.

ILACHINSKI, A. Cellular automata: a discrete universe. River Edge: World Scientific, 2001. 808 p.

KANGAS, A.; MALTAMO, M. Calibrating predicted diameter distribution with additional information. Forest Science, Bethesda, v. 46, n. 3, p. 390-396, 2000.

KESSLER, D. A.; LEVINE, H.; REYNOLDS, W. N. Coupledmap lattice model for crystal growth. Physical Review A, London, v. 42, n. 10, p. 6125-6128, 1990.

LEITE, H. G.; OLIVEIRA, F. H. T. Statistical procedure to test the identity of analytical methods. Communications in Soil Science and Plant Analysis, New York, v. 33, p. 1105-1118, 2002.

LINDEMAYER, A.; ROZENBERG, G. Automata, languages, development. Amsterdam: North Holland, 1976. 529 p.

MATHEY, A. H.; KRCMAR, E.; TAIT, D.; VERTINSKY, I.; INNES, J. Forest planning using co-evolutionary cellular automata. Forest Ecology and Management, Amsterdam, v. 239, n. 3, p. 45-56, 2007. 
MURTHY, D. N. P.; XIE, M.; JIANG, R. Weibull models. New York: J. Wiley, 2004. 396 p.

NELSON, T. C. Diameter distribution and growth of loblolly pine. Forest Science, Bethesda, v. 10, n. 1, p. 105-114, 1964.

NEUROFOREST. Disponível em: $<$ http://neuroforest.ucoz. com/>. Acesso em: 1 jun. 2012.

NOGUEIRA, G. S.; LEITE, H. G.; CAMPOS, J. C. C.; CARVALHO, A. F.; SOUZA, A. L. de. Modelo de distribuição diamétrica para povoamentos de Eucalyptus sp. submetidos a desbaste. Revista Árvore, Viçosa, v. 29, n. 4, p. 579-589, jul./ ago. 2005.

NORD-LARSEN, T.; CAO, Q. V. A diameter distribution model for even-aged beech in Denmark. Forest Ecology and Management, Amsterdam, v. 231, p. 218-225, 2006.

PALAHÍ, M.; PUKKALA, T.; BLASCO, E.; TRASOBARES, A. Comparison of beta, Johnson's SB, Weibull and truncated Weibull functions for modeling the diameter distribution of forest stands in Catalonia, north-east of Spain. European Journal of Forest Research, Georgetown, v. 126, n. 4, p. 563-571, 2007.

PHIPPS, M. J. From local to global: the lesson of cellular automata. In: DEANGELIS, D. L.; GROSS, L. J. (Ed.). Individual-based models and approaches in ecology: populations, communities and ecosystems. New York: Chapman and Hall, 1992. p. 165-187.

SEGEL, L. A. Immunology viewed as the study of an autonomous decentralized system. In: DASGUPTA, D. (Ed.). Artificial immune systems and their applications. Berlin: Springer-Verlag, 1999. p. 65-88.

SIIPILEHTO, J.; SARKKOLA, S.; MEHTÄTALO, L. Comparing regression estimation techniques when predicting diameter distributions of Scots pine on drained peatlands. Silva Fennica, Helsinski, v. 41, n. 2, p. 333-349, 2007.

SILVA, M. L. M. Estimação do diâmetro sem casca e diâmetro do cerne para árvores de Tectona grandis Linn. utilizando redes neurais artificiais. 2008. $47 \mathrm{f}$. Monografia (Graduação Engenharia Florestal) - Universidade Federal de Viçosa, Viçosa, 2008.

SOARES, T. S.; LEITE, H. G.; VALE, A. B.; SOARES, C. P. B.; SILVA, G. F. Avaliação de um modelo de passo invariante na predição da estrutura de um povoamento de Eucalyptus sp. Revista Árvore, Viçosa, v. 31, n. 2, p. 275-283, mar./abr. 2007.

ZHANG, L.; LIU, C. Fitting irregular diameter distributions of forest stands by Weibull, modified Weibull, and mixture Weibull models. Journal of Forest Research, Ottawa, v. 11, p. 369-372, 2006.

Recebido: 24 de fevereiro de 2011; aceito: 26 de junho de 2013.

Cerne, Lavras, v. 19, n. 4, p. 677-685, out./dez. 2013 
